incorrectly-i.e., because there is something at fault in the machinery-but because he has acted wrongly-i.e., in a way contrary to the dicta of the vis major, the society standard.

A piece of machinery goes on acting according to its construction for a long time, and it seems to us at the time to be perfect and complete; all at once it breaks down and stops; the process is a perfectly natural and correct one ; the wrong action would have been if it had continued to go on under circumstances which are incompatible with progression. So is it with mental processes; we legislate for perfection, not remembering that the machinery is as yet imperfectly developed for perfection. In some cases the materials are of inferior quality and they last only for a short time, but the action is perfect in its kind as far as it goes, and when it fails, it fails in accordance with what it ought to do; in other cases there are no materials for elaboration, the machine cannot be raised towards the ideal; it acts in a correct and certain way but not with the perfection which we expect, and it fails because we expect what is for it impossible. In others the action of the machine seems perfect but it does noi stop when it should, and in its unchecked working it offends the social system-then this action is called "criminal," whilst all the time the con ditions under which it was running were not perceived and ought to have been arrested, though the process was an entirely correct one and was working itself out in the only possible way.

Let us, then, understand the "criminal" process. It is not irregularity of action: it is the only possible action. We must regard the elements of the act, the factors, and the only way to be fair to the individual is to stop him when we notice something in the mechanism which is bound to work out in a way full of what the social majority deems to be danger to itself, or else to let things go on in their inevitable outworking and then, regardless of the individual, protect itself in the way which seems to it to be the best, recognising that the laws of matter are not always compatible with the ideals of mind which are artificially set up. For some natures it is the right thing to kill, for others it is the right thing to steal-i.e., no other result is possible. When society gets hold of people who act in this way it sup. presses them for the time being or else it eliminates them. There are too many weeds to have all exotics, but the weed is still a natural product.

To recapitulate. My position is that in criminal acts the mental process introduces no fresh element. If in the insane a criminal act occurs the only fresh element is the false motive, which, however, acts in the same way as does a normal one. The constituents of the voluntary process are the same in kind as in normal minds : motive, desire, feeling, end in view, action on presentation (otherwise impulse), action upon reflection-all may occur in the same way as in normal thought processes. The only difference is not in the mechanism of the elements but in their nature; thus the motive may be a wrong one (i.e., against the accepted obligations of society) or the element of feeling may be an irregular one due to structural conditions, but we must remember that just as social systems are temporary-are themselves in a mutable condition-so are mental processes. We have not in the one the perfection of organisation, though such as it is it acts in accordance with its construction neither have we in the other any but a temporary adaptation, though such as it is it acts in the way we should expect it to $d o$, and in this expectation we are rarely disappointed.

The limit of time prevents more than this brief statement of what I venture to think is an important fact to insist on and one which is not usually borne in mind-viz, the similarity of the process in criminal and legal acts. I am not discussing the question of how far the motives affect responsibility nor how far the explosiveness of feeling may be an exculpating factor. I just ask the indulgence of my medical cunfrites in insisting upon a point which by some may be considered self-evident, but which perhaps may be deemed worthy of categorical statement and of official reception or rejection.

Harley-street, W.

Royal College of Physicians of IRELand.At the monthly meeting of the President and Fellows of the Royal College of Physicians of Ireland held on Nov. 1st Dr. Charles Jacobs Tillekeratne was admitted a Licentiate in Midwifery of the College.

\section{CHRONIC INTESTINAL OBSTRUCTION DUE TO ADHESION OF A UTERINE FIBROID TO THE MESENTERY.}

\section{BY ALBAN H. G. DORAN, F.R.C.S. ENG.,} SURGEON TO THE SAMARIIAX TRRE HOSPITAL, LONDON.

A PATIENT with a small, empty umbilical sac and a lobulated uterine fibroid tumour suffered for a year from symptoms of obstruction the source of which could not be determined. When the abdominal cavity was opened the symptoms were found to be due to an adhesion of one lobe of the tumour to the mesentery, forming an arch under which coils of small intestine became incarcerated. The tumour was also remarkable for its relations to the left broad ligament. Such were the features of interest about the case which I will now relate in detail.

A woman, aged 50 years, came under the care of $\mathrm{Mr}$. F. B. Leslie Moore of Harlesden in the summer of 1906 for an attack of severe abdominal pain, tenderness, distension, and vomiting. She had been married for 25 years and had borne two children. Her last pregnancy ended when she was 31 years of age. There was a small umbilical hernia which apparently consisted of nothing more than an empty sac. The bowels could be freely opened and the symptoms subsided and disappeared after the patient had kept in bed for a few days. The attacks, however, repeatedly recurred at intervals of from two to four weeks. Mr. Moore, on examining her at the end of June, 1907, detected an abdominal tumour which was clearly a lobulated uterine fibroid. She was admitted into my wards in the Samaritan Free Hospital on July 8th.

On admission the patient was stout and pale. There was a large varicose ulcer on the inner side of the lower third of the left leg. The urine was scanty; it contained pale-red urates and a distinct trace of albumin; its specific gravity was 1020 . The patient was not allowed to get up for a few days; by the end of that space of time the daily excretion of urine exceeded 30 fluid ounces and the albumin had disappeared. The abdomen was extremely distended, measuring 41 inches in girth at the unbilical level, yet it was not tense. The integuments were pale and the subcutaneous veins were not dilated. There was a small protrusion at the umbilicus not one inch in diameter. The integuments were red; the neck of the sac admitted the tip of the forefinger and was apparently free from any adberent omentum. The sac seemed quite empty. The abdomen was tender to the touch and a bilobed mass, not freely moveable, occupied the hypogastrium. On bimanual palpation the uterus was found to be continnous with the mass which did not extend downwards below the brim of the pelvis. The periods were irregular, appearing within less than three weeks' interval; the show was more free than before the illness, but there had never been any attacks of severe hæmorrhage. The significance of the attacks of pain, tenderness, distension, and vomiting remained uncertain. There was no obstruction to the passage of flatus and fæces. The patient lived in dread of a recurrence of the symptoms.

I operated on July 16th. The tumour was bulky and solid and could not be delivered until the abdominal incision had been prolonged into the hernial sac. Then I found that the right lobe was completely separate from the left and that its uppermost part adhered posteriorly to about four inches of mesentery, close to intestine. That portion of intestine was in no way obstructed, but under the arch formed by the adhesion were several coils distinctly incarcerated. They were drawn out without any difficulty. The cause of the symptoms of obstruction was now evident. There was no other adhesion, parieta], omental, or visceral. The vessels running from the mesentery to the tumour at the point of adhesion were large and the adhesion itself was nearly one inch broad in the middle. I dissected up a thin layer of the serous coat and subjacent capsule of the tumour around the adhesion and secured it with sutures ; the fibroid was then severed from the mesentery without any loss of blood. The right Fallopian tube and ovary lay quite free from the tumour which had pushed them down into the pelvis. The right ovarian vessels were ligatured and divided; the right round ligament was moderately hypertrophied and required to be ligatured on both sides before division. A Doyen's clamp was applied to the right broad ligament which was then divided. The left lobe, or, more correctly 
speaking, the left tumour, formed a collection of solid and cystic lobules, the cysts being, at least in part, obstructed and dilated lymphatics. The left ovary lay completely below this tumour; the relations of the parts were made clear when it was found that the left Fallopian tube ran over the surface of the tumour. An extremely odematous body occupied the outer limits of the tumour in the left iliac fossa. It proved to be the left infundibulo-pelvic ligament with dilated lymphatics and greatly engorged veins. It was ligatured and divided. The left round ligament was distinctly hypertrophied ; raising a deep fold from the anterior layer of the broad ligament in which lay a fibroid of the size of a filbert, this was enucleated and then the round ligament was ligatured and divided. The left broad ligament and the serous coat of the anterior surface of the uterus were divided anteriorly, horizontally, and continuously with the line of division of the right broad ligament. The uterine vessels were now secured without difficulty; the left artery was greatly dilated. Then the base of the entire left tumour was enucleated from the lower part of the left broad ligament and the uterus was amputated above the cervix, the stump being treated in the usual manner. Lastly, I dissected away the small umbilical sac. It was absolutely empty and there were no adhesions to the neck either within or behind it. Convalescence was retarded by an attack of parametritis in the base of the left broad ligament where the connective tissue had been unavoidably bruised during the enucleation of the left tumour,

FIG. 1.

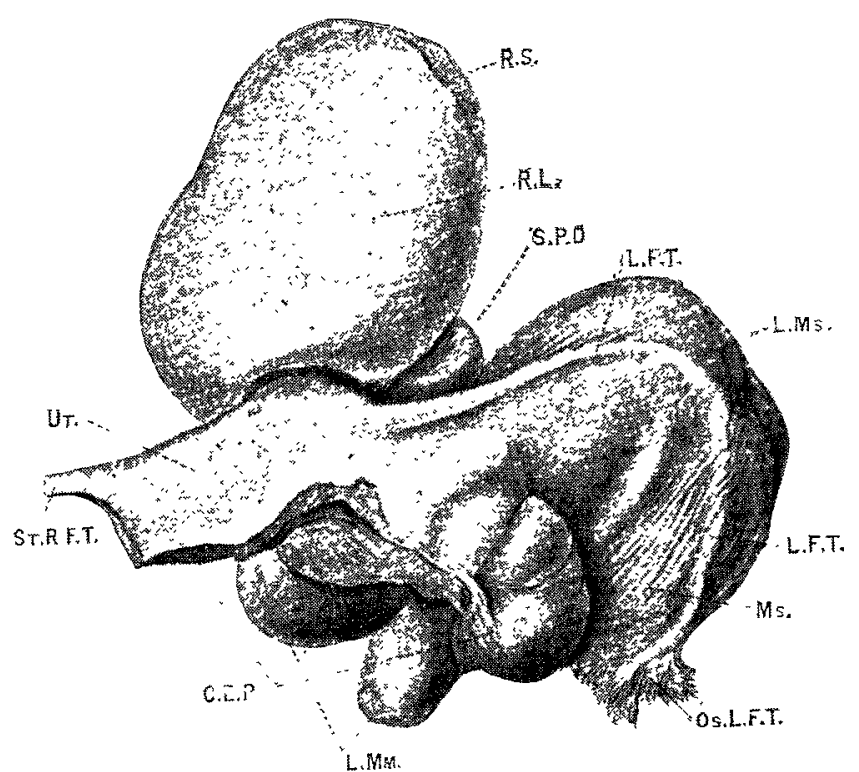

Uterus and fibroids removed by operation, sean from in front. At the summit of the right lobe there is a raw surface which adhered to the mesentery and was the cause of partial intestinal obstruction. The relations of the left lobe are also displayed. The outer part of the mesosalpinx has not been opened up by the tumour. Below lie some lobes enucleated from the mesometrium or lower portion of the broad ligament. The edge of the peritoneum divided during the amputation of the parts is also indicated. The ovaries were removed separately from 列 R.S., raw surface. R.L., right lobe. S.P.D., small pedunculated lobe. L.F.T., left Fallopian tube. L.Ms.. lobe in mesosalpinx. Ms., mesosalpinx unopened.' OS L.F.T., ostium left Fallopian tube. L.MM., lobes in mesometrium. C F.P., cut edge of peritoneum. ST R.F.T., stump right Fallopian tube. UT., uterus.

but on returning home the patient soon regained her strength. The tumour, which weighed 2 pounds 14 ounces, is preserved in the Museum of the Royal College of Surgeons of England. The appended illustrations represent its appearances very accurately, so that a lengthy pathological report would here be superfluous.

With regard to the symptoms of obstruction, the acute attacks of pain were in every instance associated with four of the symptoms observed in intestinal obstruction and in every instance two other cardinal symptoms were absent. Flatus always passed and the bowels could be opened without difficulty when the patient took to her bed, no doubt because rest in the recumbent posture allowed the incarcerated bowel to set itself free. The small umbilical sac felt empty, but touch alone was insufficient for diagnosis, and we could not make sure that there was not some condition favouring obstruction in the peritoneal cavity adjacent to the neck of the sac. There was a tumour in the lower part of the abdomen and it was correctly diagnosed. But uterine fibroids rarely obstruct any part of the intestine even indirectly, and in this case it is hard to see how the nature of the obstruction, in which the fibroid was directly concerned, could have been diagnosed. When the abdominal cavity was opened the nature of the obstraction, which could not have been determined by the clinical history or by touch, became evident to the eye. Without doubt several coils of small intestine were partially obstructed in the manner above described. In this case the essential feature was the unusual variety of obstruction which com. plicated it. It is not necessary for me to discuss intestinal obstruction in full, but I will add a few observations on its cause in the instance here recorded.

As to uterine fibroids and adhesions, experience has amply shown that fibroid tumours of the uterus do not often contract adhesions to adjacent parts, a fact of some interest when we remember how frequently we meet with that complication in association with ovarian new growths, especially dermoids. I summed up my own experience of adhesions in my third Harveian lecture on Fibroids of the Uterus and its Ligaments considered from a Clinical and Surgical Standpoint. ${ }^{1}$ We are here concerned with adhesions which are more or less liable to cause obstruction. Intimate, broad, and old-standing connexions between the omentum and the surface of a large fibroid are mainly interesting to

FIG. 2.

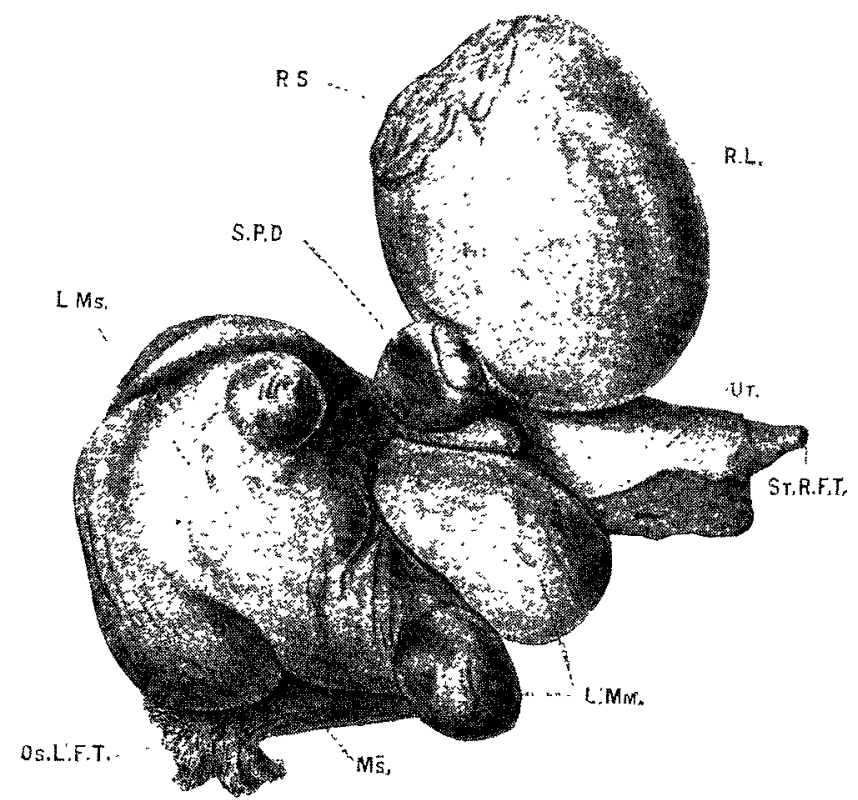

Posterior view of the same specimen; lettering as in Fig. 1. It shows the whole of the raw surface which adbered to the mesentery.

the surgeon on account of the large size which the omental vessels attain, but this condition does not tend to cause obstruction. When inflammatory disease of the Fallopian tubes and ovaries is present as a complication of uterine fibroid disease pelvic adhesions are, in my experience, the rule, but obstruction does not occur. In some instances the base of the fibroid is adherent by inflammatory bands to the sigmoid flexure or rectum; I recently met with this complication in a pregnant subject and managed to remove the tumour which was of the subperitoneal type without interrupting the pregnancy, but I could find no evidence of obstruction. We might expect otherwise when a narrow piece of omentum, often drawn oat to a cord.like process an inch or more in length, has become attached to a fibroid. Nevertheless I find from my notes that I have several times recorded this condition in patients who had never suffered from any symptoms indicating obstruction. It is the same, according to my experience, with adhesions of appendices epiploicæ, where a big arch is formed between the large intestine and the tumour, looking as though it were a regular ileum-trap. I shall revert to one case of interest where this condition was marked, but will first dismiss the subject of intestinal adhesions. They give great trouble to the operator, and in one case, which I have already published, the bowel infected the fibroid. Yet I have not come across an instance of

1 The Lancet, Feb. 21st, 1903, p. 495. 
obstruction even in two patients where $I$ had to deal with troublesome intestinal adhesions connecting the parietal peritoneum with the surface of the tumour, the result of a former exploratory operation.

Lastly, in two operations for fibroid I discovered a tumour almost fixed, with a prominent lobe closely resembling in form and position the right lobe or tumour in the case which I now publish. In each instance there was a firm adhesion which, as in the present case, was situated immediately posterior to the upper limits of the tumour. In one of the two cases it was an appendix epiploica, in the other several inches of ileum that adhered to the projecting lobe. Still, though the adhesion formed an arch under which intestine might readily have become incarcerated or even strangulated neither of the two patients had ever suffered from any symptoms indicating obstruction. My experience, however, at least shows that a lobe projecting prominently upwards and widely separate from the remaining part of a more or less fixed uterine fibroid is liable to contract adhesion which in one out of three cases caused intestinal obstruction. Granville-place, W.

\section{CANCER INFECTION AND CANCER RECUR- RENCE : A DANGER TO AVOID IN CANCER OPERATIONS.}

\section{By CHARLES RYALL, F.R C.S. ENG.,}

SURGEON TO THE CANCER HOSPITAL, BROMIPTON, LONDON, W.

SURGEONS who see much of cancer cases and who are frequently called on to exercise their skill in endeavouring to relieve or to cure patients of this malady have sometimes every reason to be disappointed with the results of their efforts. Perbaps an operation both ingenious and extensive is planned and carried out, the operator taking what he considers to be every due precaution to eradicate the disease. Yet in spite of all this care the patient subsequently develops that bugbear and disappointment of all surgeons - viz., recurrence. What is the explanation of this failure? We have been taught that where recrudescence takes place after a surgical operation for cancer this mis. fortune is due to an inadequate operation-i.e., one where the surgeon has failed, or was unable, to cut sufficiently wide of the disease. This cannot be the only reason in all cases and therefore we must seek for another. Now, our present knowledge of cancer teaches us that the trouble begins as a primary growth, that its elements then tend to invade the tissues around this growth, spreading their way through the neighbouring lymphatic system, and infecting the nearest lymphatic glands. Therefore, any adequate operation must entail excision of the growth and the possibly infected surrounding tissues. Hence we endeavour to cut wide of the primary disease, cutting into healthy tissues some distance around the growth and, at the same time, removing possibly infected lymphatic vessels and glands. We carry out such an operation because we know that for the success of any radical surgical procedure it is necessary to remove every vestige of the growth. Therefore, if the disease recurs somewhere near, but at the same time outside, the immediate field of operation it is only natural to infer that this recurrence is due to the surgeon having failed to cut sufficiently wide of the disease.

Again, one frequently sees cancer reappear in some distant organ, without any local post-operative recurrence: for example, cancer of the liver sometimes manifests itself after excision of a cancerous breast. In a case such as this it is only very obvious that in spite of the surgeon's efforts the disease had got beyond the bounds of surgical removal at the time of excision of the primary focus of infection and the surgeon, therefore, was unable to cut sufficiently wide of the disease. However, where the disease reappears within the field of operation, this reappearance, though sometimes, yet is not always satisfactorily accounted for by the argument of inadequate operation. Such also is the fact in those cases where, after the attempted removal of cancerous tissues, the disease not only appears locally but sometimes seems to recur with increased fury and virulence, in fact, in cases such as the latter, instead of the patient being relieved by the operation, it seems as if one had stirred up a hornet's nest, the post-operative history showing the disease recurring in and around the wound with extraordinary virulence and in an alarming extent, the virulence of such recurrences sometimes being so intense as to appear almost like some inflammatory malignant disease. As an explanation, therefore, of some cases where cancer recurs within the field of operation and likewise where it recurs there with increased virulence and extent one has to seek for another cause apart from inadequate operation. It is my conviction that such a cause is satisfactorily found and explained by the process of cancer infection or accidental infection of the wound by cancer cells at the time of operation. Our whole knowledge of the disease shows how very infective is the cancer cell. In the parent growth it multiplies indefinitely, and whether it be carried by the lymph or blood streams to other parts of the body there it again displays great vitality and activity, as is manifested by giving rise to secondary growths. Therefore it is not only the cells in the parent growth which are a source of danger, but also any which escape from the same by the lymph or blood streams. This is so well recognised that all radical operations are planned and executed with a view of removing all and every fragment of cancer material, because the surgeon knows that for the success of any radical operation it is necessary to remove every vestige of the growth or vestige of infection. Should he fail to do so infection persists and recrudescence of the disease inevitably takes place.

Now, if the cells in the parent growth are such a source of cancer infection, and thereby such a source of danger that any which escape in the ordinary clinical course of the disease and become disseminated through the channels above mentioned are so capable of, and liable to, start fresh secondary cancerous growths, can there be any reason to disbelieve that cancer cells which escape at the time of operation and find their way into the open wound, are an equal source of danger? Certainly there can be no reason to disbelieve that these liberated cells are capable of becoming implanted in the wound and developing into fresh malignant growth. I am quite convinced that in carrying out radical operations for cancer this form of cancer infection is one of fairly frequent occurrence and its avoidance is therefore one of vital importance. Cancer cells may escape during operation as the result of incising or lacerating the primary growth, cutting across or tearing an infected lymphatic vessel, or even from rupture of a cancerous gland, and such cells getting into the wound are quite capable of causing, and do frequently cause, cancer recurrence. This is cancer infection, or cancer implantation, and its clinical importance with regard to the causation of cancer recurrence is, in my opinion, insufficiently recognised at the present time. I do not wish to say that many surgeons are not suspicious of or aware of this danger, but I do not believe that sufficient importance is attached to it in clinical teaching of the present time.

If one wished for further evidence as to the infectivity of the cancer cell and the readiness with which it becomes implanted one has but to look at the clinical course of the disease in the abdomen. Operators of even limited experience cannot fail to be impressed with the widespread dis. semination of the disease which is so frequently found within the peritoneum. Perhaps the primary growth is in the ovary, and yet countless secondary growths of various sizes are found widely scattered over the peritoneum, both visceral and parietal, and, in fact, the dissemination of the disease may be quite as widespread as that found in peritoneal tuberculosis. The cause of this diffuse dissemination can only be explained by the fact that the cancer cell is highly infective and capable of implantation in circumstances which are perhaps particularly favourable to its growth in the abdomen. One therefore concludes that once cancer cells become parted from the parent growth and set free in the peritoneal cavity they are carried about by the movements of peristalsis, or even by the force of gravity, or perhaps their movements are aided by the presence of ascitic fluid, which is so frequently found in these cases. Thus these cells become carried from the parent growth to other parts of the peritoneum, where they become implanted, take on active growth, and cause this widespread infection. The peritoneal cavity is not the only part of the human frame which so palpably manifests the infective nature of the cancer cell, but it is the commonest. Therefore, if these cells are capable of implanting themselves wherever they happen to rest in the abdomen one can readily see that there must also be a similar proneness for liberated cells to implant themselves in an operation wound. This readiness to implantation must have a most important bearing on cancer recurrence and the success or failure of an operation. The extent of cancer infection in the peritoneum 